\title{
Pain expression is linked to personality in horses
}

\section{Carrie Ijichi, Lisa Collins and Robert Elwood}

\begin{abstract}
Tissue damage may result in pain, inducing protective behaviour such as lameness. Because we cannot directly measure an animal's subjective experience, pain research and veterinary assessment rely on these behavioural indicators when quantifying pain. This assumes that pain expression is proportional to damage but this has not been tested in animals and ignores the possible effects of personality and coping style. First, we assessed whether lameness accurately predicted the severity of tissue damage, or whether there is variance in how stoical individuals are. An experienced equine veterinarian scored horses for lameness and then the severity of tissue damage using either x-ray or ultrasound during the course of normal diagnostics in a clinical setting. Contrary to assumptions, we found no relation between scores for lameness and severity $(\mathrm{p}=0.28)$. Consequently, "Stoicism" was calculated as severity score minus lameness score. Subsequently, we tested hypotheses founded on previous work concerning how personality would be expected to link with Stoicism and pain behaviour. Personality was quantified using a validated questionnaire, completed by owners. Owners also gave their subjective opinion on how tolerant the horse was to pain using a 1-5 likert scale. This is the first paper to assess the relationships between pain behaviour and personality in animals. We found that Neuroticism is negatively related to "Stoicism" ( $\mathrm{p}=0.04)$ whereas Extroversion is positively related to levels of lameness $(\mathrm{p}=0.03)$, which may mean that pain in more easily identified in highly extrovert individuals. Future work to clarify these findings and their major implications for accurate assessment of damage and pain in animals are discussed.
\end{abstract}

KEYWORDS: Pain; Personality; Extroversion; Neuroticism; Lameness; Coping 
4 Because we cannot directly assess the affective states of animals, welfare assessment relies on physiological and behavioural indicators (Harding et al., 2004; Mendl et al., 2010). However, there are consistent individual differences in physiology that cause consistent differences in behaviour termed "personality" (Gosling and John, 1999) or "behavioural syndromes" (Sih et al., 2004). Therefore, personality may be confounding some of our measures of welfare and, if this is the case, should be taken into account in welfare assessment. Here, we use pain as a model stressor with which to assess the effect of personality on indicators of suffering. Animal pain is "an aversive sensation and feeling associated with actual or potential tissue damage" (Broom, 2001) that alters behaviour to prevent further damage (Bateson, 1991). Thus, pain influences the internal affective state of the animal and results in behavioural and physiological changes that are routinely quantified in pain research (e.g. Mogil et al. 2000; Taylor \& Weary 2000) and to aid veterinary treatment (e.g. Holton et al. 2001; Meintjes 2012). For example, lameness is used to gauge pain severity because it is presumed to be proportional to the degree of damage and hence pain experienced (Schatzmann and Spadavecchia, 2004). However, humans show inter-individual differences in pain threshold (Chen et al., 1989; Nielsen et al., 2009), as do laboratory animals (Mogil, 1999). Further, coping style is a syndrome reflecting response to stress with individuals either adopting a proactive "fight/flight" or a reactive "freeze" response. As pain can cause stress (Mellor et al., 2000), coping style may be particularly relevant when addressing issues around individual variance in response to pain. Critically, while the proactive response results in clear behavioural expression of stress due to its focus on removing or avoiding the stressor, the reactive "freeze" response is associated with fewer behavioural indicators, yet a higher physiological stress response is found in these individuals (Koolhaas et al., 2010). In effect, suffering is likely to be more readily identified on the basis of behaviour in proactive individuals whilst more severe stress remains untreated in their reactive counterparts.

This study aimed to determine if pain behaviour is associated with the severity of damage or degeneration, and whether personality is associated with level of pain behaviour demonstrated. 
a study, they are also not subject to some of the complications seen with human subjects. For example, socio-cultural conditioning in human may influence self-reports of pain (Bates et al., 1993) with socioeconomic status, gender and ethnicity playing a role. Perhaps due to these factors, human studies of the influence of personality on pain have struggled to produce clear and consistent findings. These factors are not relevant in animal studies and so such studies may be able to produce more consistent, reliable results based on more objective ratings. Horses were used here as a model animal species for several reasons. First, coping style can be predicted in this species from scores for Extroversion using a validated personality questionnaire completed by owners (Ijichi, Collins, Creighton, et al., 2013) allowing for quick assessment of multiple animals in situ without the need to put injured animals through behavioural testing. Second, they share the traits of Neuroticism and Extroversion with humans (Costa and McCrae, 1992) and several other species (e.g. Gosling \& John 1999; Ley et al. 2009) potentiating cross-species comparisons. Finally, lameness is a common problem in horses and the locomotor system is the principally treated source of pain (Dyson and Marks, 2003). Further, it is a standardised means of quantifying pain behaviour that is a routine aspect of equine veterinary practice. Thus data may be provided by highly trained professionals without the need to experimentally induce pain.

Lameness is a crucial indicator in veterinary practice due to the limited behavioural repertoire that veterinarians can avail of in the clinical setting. However, its ability to predict actual damage has not been assessed and is notably problematic (Raekallio et al., 1997). Thus our aim was to test whether lameness predicts the severity of actual damage and, if not, whether personality may be confounding this relationship. We combined findings from earlier work (Koolhaas et al., 2010; Ijichi, Collins, Creighton, et al., 2013) to make predictions about how personality would relate with pain behaviour. These predictions follow similar patterns to those recently proposed for expressing suffering in suboptimal welfare conditions (Ijichi, Collins, and Elwood, 2013). We treated Neuroticism and Extroversion as two axes working together to predict stress behaviour. Neurotic traits include a predisposition towards anxiety, a lack of emotional stability and increased stress sensitivity. Behaviour testing has shown that it relates to reactivity and avoidance of potential threats in horses 
57 (Ijichi, Collins, Creighton, et al., 2013). Extroversion includes traits such as adventurousness and 58 excitability and predicts proactive traits such as boldness in response to novelty and increased restraint resistance (Ijichi, Collins, Creighton, et al., 2013). Thus we predicted that Neuroticism would relate to the level of severity at which a behavioural response is induced, as measured by "Stoicism" and "Tolerance", but that Extroversion would relate to how the individual expressed this stress with regards to the degree of lameness behaviour. We would expect reactive/introvert animals to be more inhibited in their behavioural expression. In addition, we predicted that owner derived Tolerance scores would correlate positively with Stoicism as it had been shown that carers can grade equine pain more effectively than vets (Wilson, 2006), perhaps due to increased familiarity or having more behavioural indicators to rely upon over a longer observation period.

\section{Method}

Twenty-one horses, aged between 3-18 years old (mean $\pm \mathrm{SE}=9.4 \pm 4.2$ years) presented at an equine veterinary clinic with pre-existing lameness and were examined for lameness and damage by an equine veterinarian (H.S., MVB, MRCVS, FEI) with 32 years experience, who was blind to subject's personality scores.

\subsection{Clinical Scoring}

Clinical Lameness was assessed and scored as is common in normal clinical procedures (Adams and Stashak, 2011) using the AAEP scale of 0-5 with .5 intervals if necessary (see appendix). Several horses with a lameness score of 0 were included. This was because either the severity of their condition was being re-examined following treatment or they presented with an indicator such as swelling that suggested damage, despite a lack of lameness. The area of damage was localised using nerve blocking and the severity of damage assessed using x-ray or ultrasound depending on whether the damage was skeletal or soft tissue.

In addition to these normal clinical procedures, severity of damage was scored based on the level of lameness expected to result from such damage, as quantified using the AAEP scale. 0 indicated that no damage was present and 5 indicated significant damage that would be expected to cause severe 
pain, even at rest. Only horses that scored 1 or greater for severity and, in the cases where the cause was not immediately apparent, ceased limb-guarding following nerve-block, were included in the study. This precluded cases where lameness resulted from an unidentified secondary source. For bilateral conditions, each affected leg was separately examined for lameness (with the aid of nerve blocking) and severity. In these cases, the average score of the affected limbs was used in analysis. Stoicism was calculated as: Severity score - Clinical lameness score. Stoicism could therefore range between -5 and +5 , though due to the study inclusion restrictions, the range was between -4 and +5 .

\subsection{Owner Ratings}

Owners of suitable cases were approached and asked to complete a previously validated subjective questionnaire (Ijichi, Collins, Creighton, et al., 2013), which was used to assess the Neuroticism and Extroversion of the subjects. Owners also rated how tolerant they thought their horse was to pain in general, on a scale from 1 ("not at all tolerant") to 5 ("extremely tolerant").

\subsection{Analysis}

All analyses were conducted using "R" (R Development Core Team, 2008). Shapiro-Wilk tests revealed that data were not normally distributed, thus non-parametric tests are used throughout. Principal Component Analysis (PCA) was used to ascertain orthogonal interrelationships between variables to aid direct comparison with predictions. Data included both continuous and collapsed ordinal variables and so the PCA was based on a heterogeneous correlation matrix, consisting of Spearman's rank, polychoric and polyserial correlations. Because Stoicism is derived from Severity and Clinical Lameness they could not all be examined in one analysis, hence two PCAs were conducted. The first explored relationships between Neuroticism, Extroversion, Clinical Lameness and Severity. The second explored relationships between Neuroticism, Extroversion, Stoicism and Tolerance. Significant loadings were those above +.6 or below -.6 (Frey and Pimental, 1978). The PCAs were conducted on an acceptable sample size, albeit at the lower end of recommendations of five times as many subjects as variables (Hatcher, 1994; Bryant and Yarnold, 1995). Further, relationships between variables may be artefacts of other relationships (A loads with B but only 
because they both load with C). Therefore, post hoc Spearman rank correlations were used to confirm all relationships identified from PCA. Where testing specific predictions based on patterns from previous publications (Wilson, 2006; Koolhaas et al., 2010; Ijichi, Collins, and Elwood, 2013; Ijichi, Collins, Creighton, et al., 2013), 1-tailed tests were used.

\section{Results}

\subsection{Principal Component Analysis}

The first PCA extracted two dimensions accounting for $89.9 \%(\mathrm{~N}=21)$ of total variance (Table 1). Dimension one accounted for $62 \%$ of the variance and loaded negatively for Neuroticism and positively for Severity. The second dimension accounted for $27.9 \%$ of the variance and loaded negatively (though just under the 5\% significance threshold) for Clinical Lameness and significantly for Extroversion. The second PCA accounted for $90.2 \%$ of total variance $(\mathrm{N}=20)$ and comprised two dimensions (Table 2). Dimension 1 accounted for $69.7 \%$ of total variance. Neuroticism had a strong tendency to load positively, whilst Stoicism and Tolerance had a strong tendency to load negatively. The second dimension accounted for $20.5 \%$ of total variance and loaded negatively for Extroversion only.

\subsection{Spearman Rank Correlations}

Clinical Lameness and the Severity of the underlying condition did not load together in PCA1 and were not correlated ( $r_{s}=0.21, N=21, P=0.275$, two-tailed). Spearman correlation confirmed that Extroversion was significantly positively correlated with Clinical Lameness $\left(r_{s}=0.4, N=21, P=\right.$ 0.033 , one-tailed) but not Severity $\left(r_{s}=0.15, N=21, P=0.52\right.$, two-tailed). Neuroticism was significantly negatively correlated with Stoicism $\left(r_{s}=-0.4, N=21, P=0.038\right.$, one-tailed $)$, and showed a possible negative correlation with Tolerance $\left(r_{s}=-0.32, N=21, P=0.082\right.$, one-tailed), but not with Severity $\left(r_{s}=-0.3, N=21, P=0.181\right.$, two-tailed). Tolerance showed a possible positive correlation with Stoicism ( $r_{s}=0.32, N=21, P=0.066$, one-tailed).

\section{Discussion}


We assessed whether lameness predicted the severity of tissue damage but found no relation between the two. Lameness is expected to relate to the severity of the condition because severe conditions should result in greater pain (Schatzmann and Spadavecchia, 2004). To our knowledge this is the first time that this assumption has been tested and our findings have major implications. Pain behaviour, such as lameness, is used to assess the progression of a condition, the efficacy of analgesia and to inform decisions on humane end points (Ashley et al., 2005). Here, pain behaviour caused both over and under-estimation of severity. Over-estimation, or false-positive, is likely to result in excessive pain relief with concomitant side-effects, unnecessary invasive treatment and euthanasia. Underestimation, or false-negative, will result in unmitigated pain causing suffering, distress and detriment to health. However, links between pain behaviour and personality suggest that this may not be the result of "random noise". In both PCAs, Neuroticism and Extroversion acted as separate dimensions adding support to our hypothesis that they would act independently (Fig.1). Further, pain behaviour loaded in a manner suggestive of an influence of personality on pain related variables.

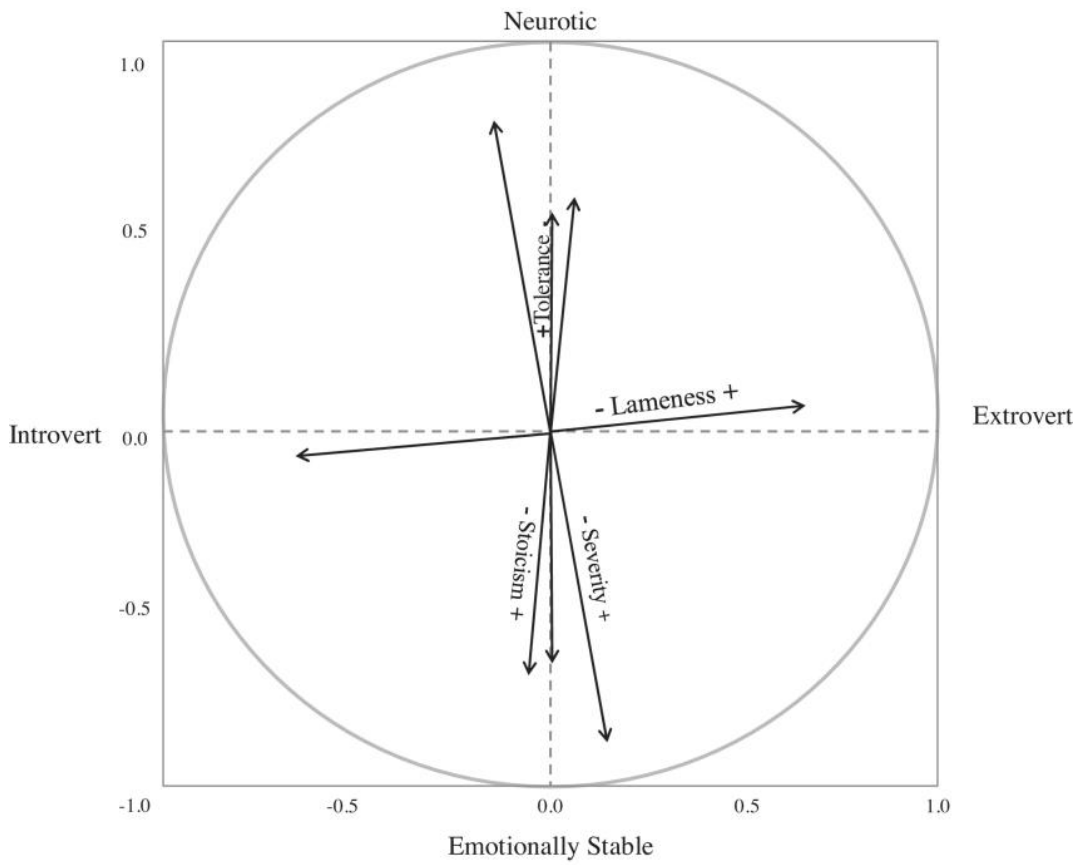

Fig 1. Diagram adapted from two PCA outputs to illustrate the relationships between variables and personality factors. The length of the arrow represents the approximate strength of the loading on both dimensions whilst its direction denotes its relationship with the personality axis. NB: arrows do not necessarily reflect relationships between pain variables. 
152 Neuroticism was found to link with several aspects of pain behaviour. First, highly neurotic horses 153 were found to be significantly less stoic as calculated from clinical data. This suggests that 154 behavioural indicators of pain are induced at a lower threshold of severity in highly neurotic animals. Second, this finding was supplemented by a tendency for them to also be rated less tolerant to pain by their owners. Although Stoicism could only be calculated from lameness scores in a clinical setting, owners were free to draw upon a wide range of contexts and behavioural changes when judging Tolerance. Evidence that this owner rating may be a useful and reliable source of information can be seen in the tendency for more tolerant horses to also be more stoical as derived from clinical data. Although pain thresholds were not explicitly tested here, these correlations support predictions that highly neurotic horses may have a reduced threshold for pain, meaning that coping responses are more easily induced. This also fits with findings of how Neuroticism affects pain responses in humans (Jones et al., 2003; Damme et al., 2004; Goubert et al., 2004).

Somewhat surprisingly, Neuroticism was negatively associated with the severity of the condition, although this was not confirmed post hoc. Clearly, personality was not expected to affect how seriously a limb was damaged or had degenerated. However, horses that are more neurotic and less stoical are likely to be more stressed by pain and therefore more effective at eliciting treatment than tolerant horses. Therefore, the severity of their condition might be lower because they were assessed at an earlier stage of degeneration or were prevented from further injury by timely diagnosis. Further, neurotic horses may have a greater fear of movement and re-injury, as seen in humans (Goubert et al., 2004), which would protect them from further damage. This suggests that being stoical has a negative impact on domestic horses in direct contrast to the benefits it provides wild counterparts if predators preferentially select vulnerable prey (Knopff et al., 2010). Further research to confirm or reject this association would be worthwhile.

On a separate axis, lameness positively associated with Extroversion. Extrovert horses did not present with more severe conditions. Though this resulted in more overt expressions of suffering, this does not demonstrate increased suffering per se but a more readily induced behavioural response to this negative affective state. The implications are that horses with low Extroversion scores are unlikely to 
clearly express their suffering and will only be mildly lame. As the assessment of mild lameness is particularly problematic (Keegan et al., 2010), introverts may go undiagnosed. In previous work, we compared behaviour during a "Bridge test", in which the horse was led across a potentially aversive large tarpaulin, to personality scores (Ijichi, Collins, Creighton, et al., 2013) . We found that Extroversion did not relate to how stressed an individual was by the test but significantly predicted the manner of the expression of stress. In this instance, horses with high Extroversion scores had an exaggerated response with obvious behavioural indicators that they found the test aversive such as rearing and escape attempts. By contrast, introverts appeared unstressed due to their passive refusal and lack of response to stimuli. Thus our current results concur with these previous findings. However, the limitations of the clinical setting mean that we only know that introverts do not become as lame. We do not know what strategies they use as part of their reactive response and this is critical if we are to accurately identify and understand pain and suffering in this personality type.

\section{Conclusions}

In this study, lameness was not a reliable indicator of the severity of damage or degeneration in horses, something anecdotally reported by some veterinarians and owners. This is the first time the relationship between severity and pain behaviour has been explicitly tested and has implications for welfare assessment, veterinary practice and pain research, all of which use pain behaviour to quantify pain. However, this finding is based on the assessment of one veterinarian, albeit one of considerable experience. Therefore, further work is required to assess whether this finding generalises across multiple raters as there will likely be some variation between raters. Further, a validated scoring system for radiographic findings has become available since the completion of this study (Lepeule et al. in press) which may prove useful for severity ratings. In addition to findings that lameness may not be a reliable indicator of severity, we provide preliminary evidence that personality plays a role in the expression of animal pain providing a spring board for more explicit testing of the links between personality and pain. For example, it would be interesting to use statistical moderator models; however this would require considerably larger sample sizes and was therefore beyond the scope of the present study. This would provide more quantitative evidence for how much of an influence 
personality has and whether the predictive value of lameness examinations can be corrected and improved by incorporating personality scores. This should be complemented by studies away from the clinical setting to ascertain what the reactive behavioural response to pain is so that critical indicators of suffering in this personality type can be identified. In addition, behavioural experiments that measure the affective dimensions of pain experience would be useful in exploring whether personality is affecting suffering as well as the behavioural response to pain.

Acknowledgements

We thank the Department for Education and Learning for funding this research. We are indebted to the staff and clients at Tullyraine Equine Clinic and in particular, to Hugh Suffern (MVB, MRCVS) for his expert rating and provision of suitable cases. Hansjoerg Kunc, Mary Montague and Kirsty McLaughlin provided invaluable comments on this manuscript in addition to several reviewers. Finally, we appreciate the statistical guidance of Martin Demspter (CStat), Queen's University Belfast and George Ploubidis, London School of Hygiene and Tropical Medicine.

Concept of study: CI; Experimental design: CI, LC, RE; Data Collection: CI; Analysis: CI, LC, RE;

Drafting and editing: CI, LC, RE.

References

Adams, O.R., Stashak, T.S., 2011. Adams and Stashak's Lameness in Horses, 6th ed. John Wiley \& Sons, Chichester, West Sussex.

Ashley, F.H., Watermen-Pearson, A.E., Whay, H.R., 2005. Behavioural assessment of pain in horses and donkeys: applications to clinical practice and future studies. Equine Vet. J. 37, 565-575.

Bates, M., Edwards, W., Anderson, K., 1993. Ethnocultural influences on variation in chronic pain perception. Pain 52, 101-112.

Bateson, P., 1991. Assessment of pain in animals. Anim. Behav. 42, 827-839.

Broom, D., 2001. Evolution of pain, in: Lord Soulsby, E., Morton, D. (Eds.), Pain: Its Nature and Management in Man and Animals. Royal Society of Medicine International Congress Symposium Series, pp. 17-25. 
Bryant, F., Yarnold, P., 1995. Principal-components analysis and exploratory and confimatory factor analysis, in: Grimm, L., Yarnold, P. (Eds.), Reading and Understanding Multivariate Statistics. American Psychological Association, Washington DC, pp. 99-136.

Chen, A., Dworkin, S., Haug, J., Gehrig, J., 1989. Human pain responsivity in a tonic pain model: psychological determinants. Pain 37, 143-160.

Costa, R.T., McCrae, R.R., 1992. Revised NEO Personaliy Inventory (NEO-PI-R) and NEO FiveFactor Inventory (NEO-FFI) Proffessional Manual. Psychological Assessment Resoures, Odessea.

Damme, S. Van, Crombez, G., Eccleston, C., 2004. Disengagement from pain: the role of catastrophic thinking about pain. Pain 107, 70-76.

Dyson, S., Marks, D., 2003. Foot pain and the elusive diagnosis. Podiatry 19, 531-565.

Frey, D., Pimental, R., 1978. Principal components analysis and factor analysis, in: Cogan, P. (Ed.), Quantitative Ethology. John Wiley \& Sons, New York, pp. 219-245.

Gosling, S.D., John, O.P., 1999. Personality dimensions in nonhuman animals: a cross-species review. Curr. Dir. Psychol. Sci. 8, 69-75.

Goubert, L., Crombez, G., Van Damme, S., 2004. The role of neuroticism, pain catastrophizing and pain-related fear in vigilance to pain: a structural equations approach. Pain 107, 234-241.

Harding, E.J., Paul, E.S., Mendl, M., 2004. Animal behaviour: cognitive bias and affective state. Nature $427,312$.

Hatcher, L., 1994. A step-by-step approach to using the SAS system for Factor Analysis and structural equation modelling. SAS Institute, Inc., Cary, N.C.

Holton, L., Pawson, P., Nolan, A., Reid, J., Scott, E.M., 2001. Development of a behaviour-based scale to measure acute pain in dogs. Vet. Rec. 148, 525-531.

Ijichi, C., Collins, L., Creighton, E., Elwood, R., 2013. Harnessing the Power of Personality Assessment: Subjective Assessment Predicts Behaviour in Horses. Behav. Processes 96, 47-52.

Ijichi, C.L., Collins, L.M., Elwood, R.W., 2013. Evidence for the role of personality in stereotypy predisposition. Anim. Behav. in press.

Jones, A., Zachariae, R., Adrendt-Nielsen, L., 2003. Dispositional anxiety and the experience of pain: gender-specific effects. Eur. J. Pain 7, 387-395.

Keegan, K.G., Dent, E. V, Wilson, D.A., Janicek, J., Kramer, R.J., Lacarrubba, A., Walsh, D.M., Cassells, M.W., Esther, T.M., Schiltz, P., Frees, K.E., Wilhite, C.L., Clark, J.M., Pollitt, C.C., Shaw, R., Norriss, T., 2010. Repeatability of subjective evaluation of lameness in horses. Equine Vet. J. 42, 92-97.

Knopff, K.H., Knopff, A.A., Kortello, A., Boyce, M.S., 2010. Cougar kill rate and prey composition in a multiprey system. J. of Wildl. Manage. 74, 1435-1447. 
Koolhaas, J.M., De Boer, S.F., Coppens, C.M., Buwalda, B., 2010. Neuroendocrinology of coping styles: Towards understanding the biology of individual variation. Front. Neuroendocrinol. 31, 307-321.

Lepeule, J., Robert, C., Bareille, N., Valette, J.-P., Jacquet, S., Seegers, H., Denoix, J.-M., 2013. A reliable severity scoring system for radiographic findings in the limbs of young horses. Vet. J. in press.

Ley, J.M., Bennett, P.C., Coleman, G.J., 2009. A refinement and validation of the Monash Canine Personality Questionnaire (MCPQ). Appl. Anim. Behav. Sci.e 116, 220-227.

Meintjes, R.A., 2012. An overview of the physiology of pain for the veterinarian. Vet. J. 193, 344348.

Mellor, D., Cook, C., Stafford, K., 2000. Quantifying some responses to pain as a stressor, in: Moberg, G., Mench, J. (Eds.), The Biology of Animal Stress: Basic Principles and Implication for Animal Welfare. CABI Publishing, CAB International, Wallingford, UK, pp. 171-198.

Mendl, M., Burman, O., Paul, E., 2010. An integrative and functional framework for the study of animal emotion and mood. Proc. Roy. Soc.: B 277, 2895-2904.

Mogil, J., 1999. The genetic mediation of individual differences in sensitivity to pain and its inhibition. Proc. Natl. Acad. Sci. U.S.A. 96, 7744-7751.

Mogil, J.S., Chesler, E.J., Wilson, S.G., Juraska, J.M., Sternberg, W.F., 2000. Sex differences in thermal nociception and morphine antinociception in rodents depend on genotype. Neurosci. Biobehav. Rev. 24, 375-389.

Nielsen, C.S., Staud, R., Price, D.D., 2009. Individual Differences in Pain Sensitivity: Measurement, Causation, and Consequences. J. Pain 10, 231-237.

R Development Core Team, 2008. R: A language and environment for statistical computing.

Raekallio, M., Taylor, P.M., Bloomfield, M., 1997. A comparison of methods for evaluating pain and distress after orthopaedic surgery in horses. J. Vet. Anaeth. 24, 17-20.

Schatzmann, U., Spadavecchia, C., 2004. Significance, diagnosis and interpretation of pain. Pferdeheilkunde 20, 51-54.

Sih, A., Bell, A.M., Johnson, J.C., Ziemba, R.E., 2004. Behavioral Syndromes: An Integrative Overview. Q. Rev. Biol. 79, 241-277.

Taylor, A.A., Weary, D.M., 2000. Vocal responses of piglets to castration: identifying procedural sources of pain. Appl. Anim. Behav. Sci. 70, 17-26.

Wilson, D., 2006. Recognition of Pain, in: Doherty, T., Valverde, A. (Eds.), Manual of Equine Anesthesia \& Analgesia. Blackwell, Oxford, UK, pp. 300-302. 